\title{
Trajetória acadêmica: notas sobre os desafios do doutorado
}

\author{
Aline Vieira Malanovicz ${ }^{1}$
}

\section{Resumo}

Este trabalho tem como objetivo descrever o processo de desenvolvimento gradual de uma pesquisa de doutorado em Administração. Considera-se que o ponto de vista discente constitui uma fonte de dados reveladora com potencial para ampliar e beneficiar a reflexão sobre o tema. $O$ método utilizado aproxima-se de uma autoetnografia para o relato das experiências vivenciadas pela pesquisadora. Por isso, a pesquisa foi desenvolvida a partir de uma abordagem metodológica do tipo qualitativodescritiva. Apresenta como resultados uma descrição do planejamento e das etapas realizadas nesse processo por uma aluna deste curso, descrevendo um cenário real que permita a reflexão sobre as possibilidades de realização de pesquisas do tipo. A descrição da trajetória apresentada expôs algumas dificuldades, surpresas, incertezas, descobertas, reflexões, elaborações teóricas, além de momentos de compreensão e outros de apreensão por parte de estudantes quanto ao desenvolvimento de pesquisas do tipo. Como conclusão, é possível dizer que o planejamento da pesquisa em etapas e a produção e submissão de artigos de pesquisa contemplando os métodos e resultados de cada etapa configuraram uma possibilidade de desenvolvimento da pesquisa avaliada como bem organizada e produtiva. Espera-se que o trabalho possa oferecer alguma contribuição para outros estudantes de Administração no percurso do desenvolvimento de seus próprios trabalhos de pesquisa.

Palavras-Chave: Pesquisa em Administração; Trajetória Acadêmica; Métodos de Pesquisa.

\section{Getting a doctoral degree: some notes about its challenges}

\begin{abstract}
This paper aims to describe the process of gradual development of a doctoral research in Administration. It is considered that the student point of view is a revealing data source which has the potential to expand and benefit the reflection on the topic. The research method applied approximates an auto-ethnography for the account of the experiences lived by the researcher. Therefore, the research was developed from a methodological approach to qualitative-descriptive. Displays results as a description of the planning and the steps taken in this process by a student of the course, describing a real scenario that allows reflection on the possibilities for conducting the type research. The description of the trajectory presented exposed some difficulties, surprises, uncertainties, discoveries, reflections, theoretical elaborations, and understanding of times and other seizure by students for the development of researches like this. In conclusion, we can say that the planning of the research stages and the production and submission of research articles based on the methods and results of each stage configured a possibility of development

\footnotetext{
${ }^{1}$ Doutorado em Programa de Pós-Graduação em Administração pela Universidade Federal do Rio Grande do Sul, Brasil (2011). Tutora Orientação TCC Pós-Graduação Gestão Pública da Universidade Federal do Rio Grande do Sul,,Brasil.malanovicz@gmail.com
} 
which was evaluated as well organized and very productive. It is expected that the work may offer some contribution to other management students on the course of development of their own research.

Keywords: Research in Management; Academic Path; Research Methods.

\section{INTRODUÇÃO}

Pensar o processo de ensino-aprendizagem exige avançar na ideia de um processo construído via aprendizagem significativa, mudança conceitual e construtivismo (MOREIRA, 1999). Isso inclui docentes conscientes das formas de estimular processos cognitivos dos estudantes com estruturas analíticas que os preparem para atuar como indivíduos proativos, construindo conhecimento a partir de dados desorganizados (VILLARDI, 2009).

Assim, a percepção das dificuldades enfrentadas pelo corpo discente no percurso dos cursos de mestrado e doutorado já foi ressaltada em trabalhos acadêmicos (MORO; BRAGANHOLO; NÁCUL; FORNARI, 2005), assim como a percepção docente (MAFRA; CAPPELLE; MENDONÇA; OLIVEIRA; PAULA, 2009; CANDIDO; VIEIRA; ALVES, 2015). Neste trabalho, considera-se que o ponto de vista discente constitui uma fonte de dados reveladora com potencial para ampliar e beneficiar a reflexão sobre o tema.

Neste contexto, este trabalho tem como objetivo descrever o processo de desenvolvimento gradual de uma pesquisa de doutorado em Administração. Pretende-se apresentar o planejamento e as etapas realizadas nesse processo por uma aluna deste curso, descrevendo um cenário real que permita a reflexão sobre as possibilidades de realização de pesquisas do tipo. Espera-se que o trabalho possa oferecer alguma contribuição para outros estudantes de Administração no percurso do desenvolvimento de seus próprios trabalhos de pesquisa.

\section{POR UMA PERSPECTIVA EPISTEMOLÓGICA}

Este tópico sinaliza a fundamentação do posicionamento epistemológico deste artigo e da importância da adoção desse ponto de vista e da relevância da realização de propostas como esta. É possível afirmar que os estudos na área de pesquisa em Administração são beneficiados quando se adotam métodos de pesquisa mais indutivos (POZZEBON, 2003). 
Essa prática possibilita a análise de fenômenos do cotidiano, da perspectiva de quem os vivencia (VIEIRA; RIVERA, 2012). As pesquisas que adotam tais métodos são interpretadas como análises de processos dinâmicos em contextos reais. Estas são informações que contribuem para um aprofundamento da análise e da compreensão de processos dinâmicos das organizações (GABRIEL, 2000).

É considerado como ponto forte metodológico o fato de os pesquisadores pertencerem à área onde está surgindo a questão problemática de seus trabalhos, e do mesmo modo, os temas de pesquisa devem surgir da prática cotidiana que os pesquisadores realizam como profissionais (TRIVIÑOS, 1995).

O mesmo posicionamento epistemológico defende que a elaboração teórica é um processo de sensemaking, de produção de sentido ou interpretação sobre as ações já realizadas pelo sujeito, e a interpretação da realidade em que o pesquisador está inserido lhe permite elaborar uma teorização com base nos aspectos que identifica na sua vivência da dinâmica dos processos (WEICK, 1999).

O relato deste o processo de desenvolvimento gradual de uma pesquisa de doutorado em Administração é um objeto de estudo que parece exigir uma abordagem metodológica que permita uma descrição aprofundada do envolvimento da pesquisadora no processo.

\section{MÉTODO}

Pode ser feita uma caracterização deste estudo como uma investigação do tipo qualitativa com estratégia etnográfica (CAVEDON, 2003). O estudo também pode ser classificado como de caráter exploratório, por buscar identificar alguns aspectos ainda não explorados sobre a questão proposta (VERGARA, 2006). A pesquisa foi desenvolvida a partir de uma abordagem metodológica do tipo qualitativo-descritiva (GIL, 2007; YIN, 2005).

A natureza qualitativa desta pesquisa permite o entendimento de situações em que se necessita de uma análise tanto descritiva quanto interpretativa (GIL, 2007), utilizando procedimentos metodológicos que buscam compreender relações, causas e justificativas que possam suportar um melhor entendimento dos acontecimentos inerentes aos fenômenos sociais (CUNHA; YOKOMIZO; BONACIM, 2013). O método utilizado aproxima-se de uma autoetnografia (TEDLOCK, 2003) para o relato das experiências vivenciadas pela pesquisadora. 
A coleta de dados desta pesquisa utilizou a técnica de observação participante de experiências vivenciadas pela pesquisadora. Os dados de campo são relatados utilizando narrativas episódicas ou evidências anedóticas, que sobrepõem história de vida e memória da pesquisadora (TEDLOCK, 2003; WEICK, 1968).

Foi feita a opção pela narrativa dos resultados da técnica de observação participante porque pode auxiliar na explicitação do conhecimento sobre as interações entre as pessoas nas organizações, envolvendo a compreensão de ações humanas, e podem informar como a teoria e a prática se unem nas ações (TEDLOCK, 2003). A exposição dos aspectos relevantes usa as experiências como parâmetro da interpretação, que foi sendo construída analiticamente durante o processo de coleta e análise dos dados (LEE, 1989).

Estes procedimentos foram adotados por levar em conta os conhecimentos anteriores da pesquisadora, pois houve forte interferência desta no decorrer dos episódios. Portanto, adotou-se a estratégia etnográfica com relatos episódicos dos dados de campo, por entender que a exploração de narrativas de uma pessoa envolvida no contexto (VIEIRA; RIVERA, 2012), no caso desta pesquisadora, pode ajudar na compreensão da dinâmica desse processo.

\section{CONTEXTO DA TRAJETÓRIA (NARRATIVA)}

O doutorado que a pesquisadora cursou teve duração de 48 meses, sendo os dois primeiros anos dedicados a disciplinas, e os dois últimos, à realização de um trabalho de pesquisa (tese de doutorado) orientado por um dos professores do Programa. A pesquisadora realizou o processo seletivo em 2007, ingressando no curso em 2008. Nos pré-requisitos exigidos pelo programa de pós-graduação para a obtenção do título de doutor, incluem-se:

- a obtenção de 60 créditos de disciplinas de duas ou quatro horas semanais por semestre;

- a comprovação de proficiência em leitura de pelo menos duas línguas estrangeiras;

- a aprovação de dois artigos científicos em eventos ou revistas nacionais ou internacionais;

- a elaboração de um ensaio teórico de qualificação com a base teórica do tema de pesquisa; 
- a defesa de um projeto de pesquisa que apresente o método a ser utilizado no trabalho;

- a defesa do trabalho final (tese) perante uma banca avaliadora com professores externos.

O programa de pós-graduação recomenda que esses requisitos sejam obtidos mais ou menos nessa ordem, sendo que os dois artigos podem ser elaborados e obter aprovação a qualquer momento durante o curso, e a proficiência em línguas estrangeiras pode ser obtida previamente ao início do curso. O ensaio teórico, o projeto de pesquisa e o trabalho final têm prazos oficialmente definidos para cada turma de ingressantes, neste caso a turma de 2008.

O programa oferece bolsas de pesquisa CAPES e CNPq para os alunos optantes por bolsa e bem classificados na seleção para ingresso no curso, sem a possibilidade de acumular outra remuneração. Para os alunos bolsistas, existe também a exigência de estágio de docência de dois semestres. No caso estudado neste trabalho, a estudante ficou bem classificada na seleção para ingresso, realizou o estágio de docência, e optou por não receber bolsa, ficando com a remuneração da empresa em que trabalha como analista de sistemas. Para tanto, foi necessário conciliar as trinta horas de trabalho por semana do emprego com os horários de aulas, palestras, cursos, reuniões, orientações e estágio docência do curso de doutorado.

Para a obtenção dos créditos durante a realização das disciplinas, são exigidos: uma disciplina obrigatória de Teorias Organizacionais; um percentual de disciplinas de métodos de pesquisa; um percentual de disciplinas da área de pesquisa escolhida pelo estudante (no caso, Sistemas de Informação); e as demais disciplinas de escolha livre, podendo aproveitar créditos de outros cursos de pós-graduação stricto sensu. A pesquisadora obteve aproveitamento de dez créditos de um Mestrado em Ciência da Computação, realizado na mesma universidade com excelente avaliação da CAPES, e concluído em 2004, restando 50 créditos a cursar.

Entre as disciplinas cursadas, destacam-se Teorias Organizacionais, Introdução à Filosofia da Ciência, Métodos de Pesquisa em Sistemas de Informação, Ateliê de Pesquisa em Sistemas de Informação, Desenvolvimento de Pesquisas via Web. Alguns semestres tiveram uma carga de leituras obrigatórias de cerca de mil páginas, entre livros, artigos e outros materiais, sendo parte considerável desse material (quase metade) escrita em inglês. Várias disciplinas tiveram como trabalho de avaliação a produção de um artigo científico sobre o tema. 
A comprovação da proficiência em leitura de pelo menos duas línguas estrangeiras foi obtida logo no início do curso, com a realização de uma prova de língua alemã na própria universidade e o aproveitamento da comprovação de proficiência em leitura de língua inglesa realizada também na mesma universidade quatro anos antes por exigência do curso de Mestrado em Ciência da Computação.

O ensaio de qualificação com a elaboração da fundamentação teórica do tema de pesquisa foi elaborado pela pesquisadora com base em uma das abordagens estudadas na disciplina de Teorias Organizacionais, no caso o interpretativismo de Karl Weick. A argumentação propunha a aplicação do conjunto teórico expresso no modelo de organização como processo de Weick (1973) ao processo de entendimento compartilhado entre usuários e desenvolvedores sobre as demandas de desenvolvimento dos sistemas de informação.

A avaliação do ensaio de qualificação é realizada por dois professores, sendo um deles externo ao programa. Em prazos determinados, os professores avaliam a argumentação elaborada e propõem questões teóricas ao aluno, que deve respondê-las detalhadamente por escrito e submetê-las à avaliação dos mesmos professores em prazos determinados. O projeto de pesquisa foi elaborado pela pesquisadora com base em uma adaptação da proposta teórica elaborada para o ensaio de qualificação, mais a proposta de método de pesquisa a ser aplicado. O projeto foi defendido perante uma banca presidida pela professora orientadora e composta por três outros professores doutores: um professor do próprio programa de Administração; uma professora de outro curso (Ciência da Computação) da mesma universidade; e outra professora de Administração de outra universidade. Essa sessão de defesa permitiu que os professores apresentassem sugestões de aprimoramento do método a ser utilizado na pesquisa empírica.

É possível dizer que a leitura e a produção de artigos de pesquisa foi incentivada em todas as disciplinas cursadas. Logo no primeiro semestre do curso, foram apresentados aos alunos os principais periódicos nacionais e internacionais da área de pesquisa sobre Sistemas de Informação, e o calendário de congressos e encontros nacionais e internacionais de pesquisa da área de Administração.

Os trabalhos finais de disciplinas, caso fossem considerados muito bons pelos professores, eram destacados pelos professores para os alunos autores, incentivando-os a submeter os artigos a esses eventos e periódicos. Esse processo permitiu à pesquisadora alcançar, ainda no segundo ano do curso, o atendimento ao pré-requisito de aprovação de dois artigos científicos em eventos nacionais: um no principal evento 
nacional de Administração (ENANPAD) e outro no principal evento nacional de Engenharia de Produção (ENEGEP).

A elaboração de artigos de pesquisa requer descrição mais detalhada neste trabalho. Foi idealizado pela pesquisadora um processo de elaboração de artigos para cada etapa da pesquisa. Esse processo incluiu as fases tradicionalmente recomendadas como etapas de qualquer pesquisa, como justificativa, levantamento bibliográfico, elaboração teórica, método de pesquisa, resultados, análises e discussões. Entre as fases da pesquisa cujos resultados foram descritos em artigos submetidos a eventos, podem ser descritas as seguintes:

- Identificação de um problema de pesquisa de interesse na área de Sistemas de Informação;

- Levantamento bibliográfico de trabalhos de pesquisa e abordagens do problema escolhido;

- Descoberta e exploração de uma teoria de base para a fundamentação da proposta de Tese;

- Levantamento bibliográfico de trabalhos de pesquisa e aplicações da abordagem teórica;

- Estudo da viabilidade de aplicar a teoria ao problema de pesquisa (ensaio de qualificação);

- Elaboração de proposições teóricas que refletem a aplicação da teoria ao problema (projeto);

- Proposta de método de pesquisa para a investigação empírica a ser realizada (projeto);

- Descrição do cenário para realização do Estudo de Caso e dos critérios para sua escolha;

- Estudo de 4 casos-piloto de teste de viabilidade das proposições elaboradas em 4 projetos;

- Análise e categorização de dados coletados via entrevistas individuais no Estudo de Caso;

- Análise e categorização de dados coletados via consulta documental no Estudo de Caso;

- Análise e categorização de dados coletados via observação participante no Estudo de Caso; 
- Descrição de 10 projetos de desenvolvimento de sistemas segundo a teoria de base (10 artigos);

- Análise comparativa entre projetos previamente descritos segundo a teoria de base;

- Discussão de achados inusitados e caminhos de trabalhos futuros suscitados pela pesquisa;

- Esta descrição do processo de desenvolvimento gradual da pesquisa.

Todos esses trabalhos foram elaborados na forma de artigos científicos de acordo com a formatação exigida pelos principais simpósios e encontros de pesquisa em Administração, foram avaliados e aprovados, tendo sido publicados nos livros de trabalhos desses eventos.

A elaboração desses artigos, com formatos estruturados de acordo com as exigências dos eventos, e dentro dos prazos desses eventos permitiu à pesquisadora a pré-produção da composição tanto do ensaio teórico, como do projeto, e também da tese propriamente dita.

\section{REFLEXÃO SOBRE O CAMINHO}

Foi possível cursar o doutorado e permanecer em um emprego trinta horas por semana. A flexibilidade de negociação de horários praticada pela empresa e das chefias imediatas foi essencial para permitir a conciliação de horários. A permanência no trabalho foi importante também para a realização da pesquisa propriamente dita, pois ofereceu o cenário do estudo de caso realizado e permitiu a imersão da pesquisadora no contexto em observação.

A exigência de serem cursados 60 créditos de disciplinas (ou neste caso, 50 créditos) apresenta desvantagens relativas à quantidade excessiva de créditos exigidos e à irregularidade de oferecimento de disciplinas realmente relevantes e interessantes para o desenvolvimento do trabalho de pesquisa de cada estudante. Disciplinas experimentais da área destacaram-se como não-proveitosas, e o terceiro semestre do curso destacou-se pela ausência de oferecimento de disciplinas de interesse.

Por outro lado, algumas disciplinas, como Teorias Organizacionais, Introdução à Filosofia da Ciência, Métodos de Pesquisa em Sistemas de Informação, Ateliê de Pesquisa em Sistemas de Informação, Desenvolvimento de Pesquisas via Web, 
ofereceram muitos recursos para a realização do trabalho de pesquisa propriamente dito. $\mathrm{Na}$ maioria das disciplinas, a elaboração de artigos pelos alunos como trabalhos de avaliação das disciplinas foi considerada proveitosa para a reflexão e a produção de sentido sobre os temas estudados.

Parte considerável do material de leitura das disciplinas estava escrita em inglês, e nada escrito em alemão ou em outras línguas (apenas um único artigo em espanhol). Assim, a proficiência em uma segunda língua estrangeira não teve utilidade perceptível neste curso.

É possível esboçar uma interligação entre algumas disciplinas e alguns pré-requisitos do curso. Por exemplo, as disciplinas de Teorias Organizacionais e Introdução à Filosofia da Ciência ofereceram os fundamentos para a elaboração do ensaio teórico de qualificação. Já as disciplinas de Métodos de Pesquisa e de Ateliê de Pesquisa em Sistemas de Informação ofereceram subsídios para a elaboração e o refinamento do projeto de pesquisa.

O prazo para a entrega do ensaio teórico de qualificação coincidiu com o fim do terceiro semestre de disciplinas, resultando, na prática, em apenas um mês livre para dedicação exclusiva à elaboração da fundamentação teórica do tema de pesquisa a ser explorado na tese. O processo de avaliação do ensaio, na forma de questões teóricas propostas pelos professores avaliadores, auxiliou a pesquisadora a descrever mais detalhadamente alguns aspectos teóricos da proposta de pesquisa a ser realizada. A apresentação do ensaio para os colegas e outros professores foi considerado produtivo no sentido de destacar a necessidade de clareza, coerência e concisão da proposta de elaboração teórica da pesquisa.

Já o projeto de pesquisa teve prazo coincidindo com o fim do quinto semestre do curso, período em que todos os créditos de disciplinas já haviam sido obtidos. Desse modo, o quinto semestre foi praticamente todo dedicado à elaboração e ao refinamento do projeto. A elaboração prévia da argumentação teórica do tema de pesquisa foi considerada muito produtiva para a composição do projeto de pesquisa, pois aquela primeira parte já estava elaborada, avaliada, discutida e aprimorada, e a principal tarefa na elaboração do projeto pôde ser direcionada ao método e às técnicas e procedimentos a serem utilizados na pesquisa empírica a ser realizada.

As sugestões apresentadas pelos professores da banca avaliadora do projeto puderam ser integradas aos procedimentos que foram adotados na pesquisa empírica, e as indicações de aprimoramento também foram integradas ao documento apresentado como projeto, o 
qual depois evoluiu para compor os capítulos iniciais do documento da tese propriamente dita.

Em relação à produção de artigos de pesquisa, o planejamento da pesquisa em fases ou etapas permitiu o gerenciamento do tempo e das tarefas de um projeto de longo prazo como uma tese de doutorado, em tarefas de curto e médio prazo como a elaboração de artigos. Vale comentar a produção de cada artigo que descreve cada etapa dessa pesquisa.

Inicialmente, a pesquisadora fez a identificação de um problema de pesquisa de interesse na área de Sistemas de Informação. Dada a prática profissional da pesquisadora como analista de sistemas, o problema do entendimento compartilhado entre usuários e desenvolvedores a respeito das demandas de desenvolvimento de sistemas apresentou-se como questão prática de interesse, e o trabalho de pesquisa identificou as abordagens de artigos da área de Sistemas de Informação, Computação e Engenharia sobre o tema, identificando a preponderância de abordagens tecnicistas e funcionalistas.

A seguir, a pesquisadora desenvolveu um levantamento bibliográfico de trabalhos de pesquisa e abordagens do problema escolhido na área de Administração e, em especial, Estudos Organizacionais, com as abordagens teóricas que contemplaram o tema do entendimento compartilhado, identificando que pouco tratamento teórico foi dado ao tema, quando observado sob a ótica da área de pesquisa sobre Sistemas de Informação. O passo seguinte da pesquisadora foi a descoberta e exploração de uma teoria de base para a fundamentação da proposta de tese, o que aconteceu ao longo do curso de disciplina de Teorias Organizacionais, tendo sido identificado o interpretativismo de Karl Weick como conjunto teórico com potencial para fundamentar os trabalhos sobre o tema da pesquisa.

Logo depois, a pesquisadora realizou um levantamento bibliográfico de trabalhos de pesquisa e aplicações da abordagem teórica, identificando aplicações da teoria de base escolhida em inúmeros contextos organizacionais, mas também constatando a escassez dessas aplicações dessa teoria em contextos referentes à área de Sistemas de Informação.

Tendo identificado essa lacuna na interconexão de teorias e problemas de pesquisa, a pesquisadora realizou um estudo da viabilidade de aplicar a teoria ao problema de pesquisa. Esse trabalho teórico resultou no documento do ensaio de qualificação, avaliado pelos professores como tendo apresentado ideias promissoras para $\mathrm{o}$ 
desenvolvimento teórico da área de pesquisa em Sistemas de Informação, e do desenvolvimento das aplicações práticas do interpretativismo de Weick.

O passo seguinte, contemplando as sugestões dadas pelos professores avaliadores do ensaio, foi a elaboração de proposições teóricas que refletem a aplicação da teoria ao problema. Essas proposições associaram os elementos do modelo de organização de Weick às fases possíveis em um processo de entendimento compartilhado entre usuários e desenvolvedores, e compuseram a fundamentação teórica do projeto de pesquisa elaborado.

A seguir, a pesquisadora elaborou artigo com uma proposta de método de pesquisa para a investigação empírica a ser realizada, estabelecendo a estratégia de pesquisa como estudo de caso em determinada empresa, com acompanhamento de alguns projetos de desenvolvimento de sistemas, utilizando técnicas de entrevistas individuais, observação participante e consulta documental para coleta de dados. Esta proposta de método constituiu a parte prática do documento do projeto de pesquisa elaborado.

Considerando a proposta de estratégia de estudo de caso, a pesquisadora elaborou a descrição do cenário para realização do estudo de caso e dos critérios para sua escolha, detalhando o contexto de realização da pesquisa, o funcionamento da empresa escolhida para a investigação e o modo de produção em parceria entre área de negócio e de tecnologia (usuários e desenvolvedores) utilizado pela empresa, que é a mesma em que a pesquisadora atua como analista de sistemas e, portanto, está inserida no contexto profissional.

Antes de propor o projeto para aprovação, a pesquisadora realizou estudo com quatro casos-piloto de teste de viabilidade das proposições elaboradas. Foram selecionados quatro projetos de desenvolvimento de sistemas, que foram analisados segundo as proposições teóricas do modelo de pesquisa escolhido. A viabilidade dessa análise e descrição encorajou o prosseguimento da pesquisa e a proposição do projeto.

Tendo sido aprovado o projeto de pesquisa elaborado, a pesquisadora foi a campo e realizou a coleta de dados. Com base nos dados coletados, foram desenvolvidos três diferentes artigos com os resultados preliminares dessa coleta de dados. Nesses três artigos, foi descrita a análise e categorização dos dados coletados no estudo de caso, via entrevistas individuais, observação participante e consulta documental, sendo um artigo para cada fonte de dados. Esta foi uma etapa trabalhosa da pesquisa, mas que permitiu a depuração dos dados em prazos que facilitaram a posterior análise de maneira individualizada por projeto e também de maneira comparativa entre projetos. 
Tendo chegado ao fim da coleta de dados, foi possível para a pesquisadora realizar a análise e a descrição de cada um dos dez projetos de desenvolvimento de sistemas selecionados, utilizando a teoria de base. Foram assim elaborados dez artigos diferentes, mas assemelhados em sua forma, um para cada projeto analisado, de acordo com o modelo dado pelas proposições elaboradas. Esta fase exigiu a atenção individualizada e a imersão no contexto de cada projeto de desenvolvimento, e permitiu à pesquisadora identificar empiricamente a plausibilidade da aplicação das proposições teóricas elaboradas.

Depois da análise de cada projeto individual, a pesquisadora realizou então uma análise comparativa entre projetos previamente descritos, segundo a teoria de base. Essa comparação permitiu evidenciar aproximações e diferenças no transcorrer de cada projeto, e convergências e possíveis divergências do andamento dos projetos em relação ao previsto no modelo de base das proposições elaboradas. Esta fase da pesquisa ofereceu uma visão geral da aplicabilidade das proposições nos projetos, confirmando a sua plausibilidade.

Depois das análises individuais e comparativas dos projetos estudados, chegando ao fim do processo da pesquisa como um todo, foi possível à pesquisadora esboçar uma discussão de achados inusitados e caminhos de trabalhos futuros suscitados pela pesquisa. Foi uma etapa gratificante como coroamento de todo o trabalho, pois foi possível perceber os novos caminhos que esta pesquisa despretensiosa permitiu abrir para outros desbravadores.

E no fim de todo processo, foi possível realizar a presente descrição do processo de desenvolvimento gradual da pesquisa, descrevendo todas essas fases e sua importância. Um registro merece ser feito a respeito do apoio emocional oferecido pelos pais, irmã, namorado, colegas doutorandas, colegas de empresa e até mesmo orientadora durante o processo de realização de uma pesquisa de doutorado em Administração. Este aspecto é essencial para a manutenção da tranquilidade e para evitar o estresse real atribuído à realização de um trabalho de longo prazo como uma tese.

Além disso, a organização do tempo para as tarefas no estilo "don’t panic", "no stress" permitiu o escape para a leitura de muita literatura e de dicas e anedotas compartilhadas em sites bem-humorados sobre a vida dos pós-graduandos nos intervalos autodefinidos pela pesquisadora entre as tarefas e a composição dos artigos. 
Especial destaque de produtividade pode ser dado aos encontros entre doutorandas chamados de "sabadão da tese", ocasiões para se compartilhar progressos e dificuldades, além da celebração da amizade.

\section{CONSIDERAÇÕES FINAIS}

Este trabalho descreveu o processo de desenvolvimento gradual de uma pesquisa de doutorado em Administração. Foi possível apresentar o planejamento e as etapas realizadas por uma aluna deste curso, descrevendo um cenário real que permitiu a reflexão sobre as possibilidades de realização de pesquisas do tipo. A descrição da trajetória apresentada expôs dificuldades, incertezas, descobertas, reflexões, elaborações teóricas, além de momentos de compreensão e outros de apreensão por parte da pesquisadora quanto ao desenvolvimento de pesquisas do tipo.

Avalia-se que o mesmo método de pesquisa adotado neste trabalho, observação participante das experiências vivenciadas pela pesquisadora, poderia ser aplicado a outros contextos, com as devidas reflexões e adaptações para o percurso de outros estudantes. Isso poderia auxiliar o estudo do processo de pesquisa em outras organizações, e superar uma limitação deste estudo, que é a abordagem de um contexto único.

Como conclusão, é possível dizer que o planejamento da pesquisa em etapas e a produção e submissão de artigos de pesquisa contemplando os métodos e resultados de cada etapa configuraram uma possibilidade de desenvolvimento da pesquisa avaliada como bem organizada e produtiva. Da reflexão sobre o processo, fica a constatação de que os procedimentos utilizados permitiram à estudante a apropriação do sentido da teoria estudada e a compreensão da articulação e da importância e dos resultados de cada etapa da pesquisa, evidenciando as interligações e a coerência necessária ao processo de pesquisa como um todo.

Um aspecto da trajetória que apresentou extrema relevância para o processo como um todo foi o apoio prático e também emocional compartilhado entre a pesquisadora e suas colegas doutorandas, às quais um agradecimento é pouco para expressar a sua importância neste projeto. Espera-se que o trabalho seja capaz de oferecer contribuição relevante para outros alunos de Administração no sentido de oferecer uma sugestão de forma de organização das atividades no percurso do desenvolvimento de seus próprios trabalhos de pesquisa. 


\section{REFERÊNCIAS}

CANDIDO, R. B. ; VIEIRA, A. M. ; ALVES, L. R. . Trajetória docente: desafios no contexto brasileiro. Journal of Economic Sociology Studies, v. 5, n. 2, p. 504-517, 2015 .

CAVEDON, N. R. Antropologia para Administradores. Porto Alegre: UFRGS, 2003. CUNHA, J. A. C.; YOKOMIZO, C. A.; BONACIM, C. A. G. Miopias de uma lente de aumento: as limitações da análise de documentos no estudo das organizações. Revista Alcance, v. 20, n. 4, p. 431-446, 2013.

GABRIEL, Y. Storytelling in organizations: facts, fictions and fantasies. Oxford: Oxford University Press, 2000.

GIL, A. C. Como elaborar projetos de pesquisa. São Paulo: Atlas, 2007.

LEE, A. A scientific methodology for MIS case studies. MIS Quarterly, v.13, n.1, 1989.

MAFRA, F. L.; CAPPELlE, M. C.; MENDONÇA, M. C.; OLIVEIRA, M. L.; PAULA, M. G. Ensino-Aprendizagem numa Perspectiva Crítica. In: 33. EnANPAD, 19 a 23 set. 2009, São Paulo/SP. Anais... Rio de Janeiro, ANPAD, 2009.

MOREIRA, M. A. Aprendizagem Significativa. Ed. UnB, Brasília, 1999.

MORO, M. M.; BRAGANHOLO, V.; NÁCUL, A.C.; FORNARI, M. The Successful Grad Student. IEEE Potentials, v. 24, n. 3, p. 11-13,45, 2005.

POZZEBON, M. Criteria for Conducting and Evaluating Critical Interpretive Research in the IS Field. Cahier du GreSI. n.3, v.14, dez.2003.

TEDLOCK, B. Ethnography and Ethnography Representation. In: DENZIN, N. K.; LINCOLN, Y. S. (eds.). Handbook of Qualitative Research. 2.ed. California: Sage, 2003.

TRIVIÑOS, A. N. S. Introdução à Pesquisa em Ciências Sociais: a pesquisa qualitativa em educação (Positivismo, Fenomenologia, Marxismo). São Paulo: Atlas, 1995.

VERGARA, S. C. Métodos de pesquisa em Administração. São Paulo: Atlas, 2005.

VIEIRA, A. M.; RIVERA, D. P. B. A Hermenêutica no Campo Organizacional: duas possibilidades interpretativistas de pesquisa. Revista Brasileira de Gestão de Negócios, v. 14, n. 44, p. 261-273, 2012. 
VILLARDI, B. Q. Aprendizagem Coletiva numa IES privada: arcabouço analítico dos microprocessos de poder e emocionalidade de docentes ensino e pesquiso na graduação. In: 33. EnANPAD, 19 a 23 set. 2009, São Paulo/SP. Anais... Rio de Janeiro, ANPAD, 2009.

WEICK, K. E. Theory construction as disciplined reflexivity: Trade-offs in the 90s. Academy of Management Review, 24/4: 797-806, 1999.

WEICK, K. E. Systematic Observational Methods. In: LINDZEY,G.; ARONSON,E. (orgs). Handbook of Social Psychology, London: Lawrence Erlbaum, 1968.

YIN, R. Estudo de Casos: Planejamento e Métodos. Bookman, Porto Alegre, 2005. 